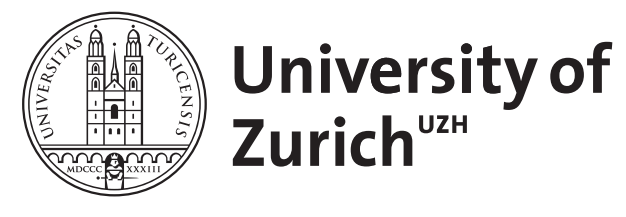

Zurich Open Repository and Archive

University of Zurich

University Library

Strickhofstrasse 39

CH-8057 Zurich

www.zora.uzh.ch

Year: 2015

\title{
Emotionen in medizinischer Kommunikation
}

Lindemann, Katrin

Posted at the Zurich Open Repository and Archive, University of Zurich

ZORA URL: https://doi.org/10.5167/uzh-139083

Book Section

Published Version

Originally published at:

Lindemann, Katrin (2015). Emotionen in medizinischer Kommunikation. In: Busch, Albert; SpracnzFogasy, Thomas. Handbuch Sprache und Medizin. Berlin, Boston: De Gruyter, 154-162. 


\title{
Katrin Lindemann \\ 8. Emotionen in medizinischer Kommunikation
}

\begin{abstract}
Emotionen wie z. B. Angst oder Ärger werden häufig als schwieriger Aspekt medizinischer Kommunikation beschrieben, der die Beteiligten vor komplexe Gesprächsaufgaben stellen kann. Zugleich kann aber, wenn der Thematisierung von Emotionen Raum gegeben wird, darin ein entscheidender Faktor für den Behandlungsprozess liegen: Das Eingehen auf Emotionen gilt im medizinischen Kontext und vielfach auch im Erfahrungswissen von PatientInnen als wichtig nicht nur für eine gelingende Kommunikation, sondern auch für die Behandlung und die Bewältigung von Erkrankungen. Gesprächsanalytische Arbeiten zeigen darüber hinaus, dass die vergleichende Untersuchung des Umgangs mit Emotionen auch differenzialtypologisch aufschlussreich sein kann; z. B. bei der interaktiv hervorgebrachten Unterscheidung verschiedener Formen von Angst, wie Alltagsangst oder Panik, die diagnostisch oft schwierig voneinander abzugrenzen sind.
\end{abstract}

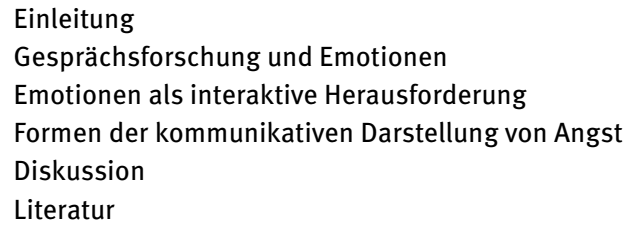

\section{Einleitung}

Emotionen bilden ein immer wiederkehrendes Element von Krankheitsdarstellungen und werden von Betroffenen im Gespräch häufig als (ein) zentraler Bestandteil des subjektiven Krankheitserlebens und -wissens relevantgesetzt. Dies gilt nicht nur für einschneidende oder sogar lebensbedrohliche Erkrankungen, sondern auch für allgemein nicht als ernsthaft eingestufte Ereignisse wie beispielsweise eine Erkältung: Die Konfrontation mit einer Beeinträchtigung des persönlichen Wohlbefindens löst häufig Ärger und/oder Angst aus, und diese Emotionen tauchen z. B. in Schilderungen des Erlebens von Symptomen, von Auswirkungen auf den persönlichen Alltag oder von Erfahrungen im Kontakt mit BehandlerInnen auf. In der Medizin gilt die Berücksichtigung von Emotionen als wichtig für eine gelungene Kommunikation mit den PatientInnen (vgl. z. B. Buser/Schneller/Wildgrube 2007, 231); das Gespräch zwischen PatientInnen und ÄrztInnen wiederum wird als zentral für den Behandlungserfolg und die erfolgreiche Bewältigung einer Erkrankung angesehen (vgl. ebd., 225, 
231). Somit bilden Emotionen einen wichtigen Aspekt medizinischer Kommunikation (vgl. auch Suchman u. a. 1997).

Der Umgang mit eigenen und fremden Emotionen stellt eine kommunikative Aufgabe für die Beteiligten dar, die potentiell zu jedem Zeitpunkt und in jedem dieser Gespräche relevant werden kann - ungeachtet der Gesprächsphase, des Interaktionstyps (z. B. Anamnese- oder Entlassungsgespräch), der Fachdisziplin oder des Behandlungsrahmens (ambulant, stationär). Allerdings machen diese Faktoren Emotionsthematisierungen unterschiedlich stark erwartbar.

Die zentrale Rolle von Emotionen spiegelt sich im Allgemeinen im konkreten Gespräch nur bedingt wider, wie eine Reihe gesprächs- und konversationsanalytischer Arbeiten gezeigt haben (zur besseren Lesbarkeit sowie aus Platzgründen wird im Folgenden „Gesprächsforschung“ als Oberbegriff verwendet): Sowohl vonseiten der PatientInnen als auch von ärztlicher Seite werden Emotionen zwar hin und wieder explizit thematisiert, häufig aber nur implizit angesprochen, lediglich mit einer Geste oder mimisch zum Ausdruck gebracht oder auch explizit als nicht relevant bezeichnet. Emotionen zeichnen sich bei genauem Hinsehen vor allem durch ihren nur angedeuteten Charakter, ihre Verneinung oder ihre offensichtliche Problematik für den Gesprächsverlauf aus.

Somit scheint es eine Spannung zu geben zwischen dem patienten- und arztseitigen Wissen um die Bedeutung von Emotionen für Krankheitserleben und -bewältigung einerseits und gleichzeitig $\mathrm{zu}$ beobachtenden interaktiven Herausforderungen andererseits.

In den folgenden Kapiteln werden unterschiedliche Facetten von Emotionsdarstellungen in medizinischen Gesprächen anhand gesprächsanalytischer Beobachtungen exemplarisch verdeutlicht: Nach einem Forschungsüberblick (Kapitel 2) werden in Kapitel 3 zunächst Beispiele untersucht, in denen die Thematisierung von Emotionen für die Beteiligten zur interaktiven Herausforderung wird. Kapitel 4 befasst sich am Beispiel der Emotion Angst mit differenzialtypologischen Erkenntnismöglichkeiten der Untersuchung von Emotionsäußerungen. In Kapitel 5 werden die Ergebnisse kurz diskutiert.

\section{Gesprächsforschung und Emotionen}

Medizinische und psychologische Perspektiven auf Emotionen legen einerseits deren Subjektivität als gegeben zugrunde, andererseits versuchen sie, dieses Erleben so objektiv wie möglich zu beschreiben. Die Gesprächsforschung interessiert sich im Gegensatz dazu nicht für Außenbeschreibungen der „inneren“ Seite von Emotionen, sondern vielmehr für deren interaktiven Charakter. Damit verlieren auch Aussagen oder Spekulationen über das „tatsächliche“ subjektive Erleben der Beteiligten ihre Bedeutung (vgl. z. B. Fiehler 2001, 1427). 
Bei der Kommunikation von Emotionen wirken zumeist mehrere Ebenen der Interaktion mit (vgl. im Überblick z. B. Sorjonen/Peräkylä 2012, 5-10): Die Beteiligten setzen sowohl sprachliche und parasprachliche Mittel ein als auch Nonverbales sowie Methoden der Gesprächsstrukturierung wie z.B. die Platzierung von Äußerungen. Zudem ist grundsätzlich zwischen zwei unterschiedlichen Formen der Darstellung zu unterscheiden (vgl. Fiehler 2001, 1430): Zum einen können Emotionen mit ganz unterschiedlichen Mitteln zum Ausdruck gebracht werden, wobei sie nicht unbedingt zum Thema des Gesprächs werden müssen, sondern auch implizit bleiben können (z. B. durch einen ängstlichen Gesichtsausdruck, der Angst kommuniziert, ohne sie explizit zu benennen); zum anderen können Emotionen explizit verbalisiert werden. Darüber hinaus kann sich die kommunikative Darstellung sowohl auf Emotionen in der aktuellen Gesprächssituation als auch auf vergangenes Erleben beziehen.

Die Untersuchung von Emotionen ist kein klassisches Thema der Gesprächsforschung, sondern rückt erst seit einigen Jahren vermehrt in ihren Fokus. (Auf einen vollständigen Literaturüberblick muss an dieser Stelle verzichtet werden. Siehe daher z. B. den Sammelband Peräkylä/Sorjonen (2012); zusammenfassend z. B. Ruusuvuori (2013), Sorjonen/Peräkylä (2012).) Zugleich formuliert aber bereits Sacks (1992) in einer Vorlesung im Frühjahr 1971 Überlegungen zum Thema „Communicating a feeling“ (ebd., 402-409), und im Frühjahr 1972 gibt er Hinweise auf typische Platzierungen von Emotionsthematisierungen (vgl. ebd., 570-575).

Auch in weiteren frühen Arbeiten werden Emotionen mehr oder weniger explizit behandelt. Hier sind insbesondere Jeffersons Untersuchungen $\mathrm{zu}$ „troubles talk“ $\mathrm{zu}$ nennen (Jefferson 1988 u.a.). Sorjonen/Peräkylä (2012) merken zusammenfassend zum allgemeinen Stand der konversationsanalytischen Untersuchung von Emotionen an:

[...] the existing research is scattered and, more important, in many studies emotion forms a subordinate analytical theme rather than being the central topic addressed. (ebd., 12)

Besondere Bedeutung haben Emotionen für institutionelle Kommunikation, insbesondere für medizinische/psychotherapeutische Gespräche. Zwar bilden Emotionen auch in Arbeiten zur medizinischen Kommunikation nur hin und wieder den zentralen Untersuchungsgegenstand, aber sie berühren - aufgrund der Bedeutung von Emotionen für Krankheitserleben und -bearbeitung - fast alle diese Untersuchungen mehr oder weniger direkt. Im Fokus gesprächsanalytischer Arbeiten zu Emotionen im Kontext medizinischer Kommunikation stehen häufig daraus resultierende interaktive Herausforderungen. Oft wird dabei auf ein Vermeiden von Emotionalität vonseiten der Ärzte hingewiesen (ausführlich dazu Kap. 3). Es werden zumeist Angst und Ärger untersucht, also die für diesen Kontext zentralen Emotionen. Während Angst zunächst als nicht weiter differenziertes Phänomen untersucht wurde, wird seit einigen Jahren der Versuch unternommen, aufgrund unterschiedlicher Formen der Emotionsdarstellung eine teilnehmergenerierte Angsttypologie zu entwickeln. Dieses 
Vorhaben beruht auf der empirischen Beobachtung, dass Angst im Gespräch nicht in immer gleicher Art und Weise kommuniziert wird, sondern dass die Beteiligten voneinander unterscheidbare Merkmalsbündel zur Darstellung verschiedener Formen von Angst nutzen (ausführlich dazu Kap. 4).

\section{Emotionen als interaktive Herausforderung}

Sowohl aus Perspektive der Gesprächsforschung als auch der Medizin gelten Emotionen als wichtiger und zugleich besonders schwieriger Aspekt von Arzt-PatientGesprächen.

In der Medizin werden Emotionsthematisierungen als hilfreich angesehen, um eine vertrauensvolle Beziehung zwischen Ärztin/Arzt und PatientIn herzustellen. Darüber hinaus soll den PatientInnen eine umfassende Krankheitsdarstellung ermöglicht werden, wozu in vielen Fällen auch Emotionen gehören, da diese einen wichtigen Teil des Krankheitswissens und -erlebens darstellen. Für eine Reihe von Gesprächen gelten Emotionen als besonders relevant, wie z. B. in der Onkologie, der Gynäkologie, der Intensivmedizin, der Chirurgie, im Umgang mit chronisch und/oder schwer Kranken oder mit Sterbenden (vgl. z. B. Buser/Schneller/Wildgrube 2007, 277315). Somit wird insbesondere im Umgang mit PatientInnen in Ausnahmesituationen auf die Bedeutung von Emotionen im Gespräch hingewiesen. Diese Relevantsetzung schlägt sich z. T. in Handreichungen zur Übermittlung negativer Diagnosen nieder (vgl. z. B. Lüdke/Langkafel 2008) sowie in Lehrbüchern zur ärztlichen Gesprächsführung, in denen allgemeine Hinweise zum Umgang mit Emotionen gegeben werden (vgl. z. B. Buser/Schneller/Wildgrube 2007, 225-258, 277-315; Rockenbauch/Decker/ Stöbel-Richter 2006, 133-168).

Die tatsächliche Gesprächspraxis spiegelt indes nicht immer das theoretische Wissen um die Relevanz von Emotionen wider. Als Erklärung dafür wird zum einen die Herausforderung hervorgehoben, angemessen auf (negative) Emotionen von PatientInnen einzugehen, insbesondere im Zusammenhang mit schweren Erkrankungen oder im Umgang mit Sterbenden (vgl. Buser/Schneller/Wildgrube 2007, 252-280). Darüber hinaus wird auf die Schwierigkeit hingewiesen, als Ärztin/Arzt eigene Emotionen zu bewältigen oder zu kontrollieren, die insbesondere in schwierigen Gesprächssituationen auftreten können (vgl. ebd., 237 f., 277-315).

Die Gesprächsforschung konstatiert einerseits eine auf Vermeidung von Emotionalität ausgerichtete ärztliche Gesprächshaltung (vgl. z. B. Beach u.a. 2005; Bergmann 1992; Fiehler 1990; 2005; Lalouschek 1993). Andererseits ist zu beobachten, dass PatientInnen Emotionen oft nicht explizit thematisieren, sondern sie nur vage, implizit (vgl. z. B. Suchman u. a. 1997, 679 f.) oder z. B. mimisch, durch eine Geste oder durch Schweigen in das Gespräch einbringen. Ein solches Thematisierungsangebot kann zum einen leicht übersehen werden - ÄrztInnen reagieren darauf häufig mit 
einer Defokussierung des Emotionalen (vgl. Beach u. a. 2005, 396-399; Suchman u. a. 1997, 680); zum anderen kann ein solches Angebot mit wenig interaktivem Aufwand übergangen werden bzw. es erfordert umgekehrt erhöhten Aufwand, das nur Angedeutete explizit zum Thema des Gesprächs zu machen. So konnte in gesprächsanalytischen Arbeiten gezeigt werden, wie die Thematisierung von Emotionen erst in einem langwierigen Prozess realisiert wird und sowohl interaktive Mühe als auch Zeit benötigt (vgl. Gülich/Couper-Kuhlen 2007; Heritage/Lindström 2012; Lindtner-Rudolph/ Bardenheuer, in diesem Band); mehr Zeit, als in vielen medizinischen Gesprächen zur Verfügung steht.

Die folgenden Beispiele stammen aus einem Gespräch zwischen einer Hausärztin und einer Patientin, die wegen Ellenbogenschmerzen die Praxis aufsucht. Die Ausschnitte zeigen, wie die Ärztin zunächst zwei Emotionsthematisierungen der Patientin nicht aufnimmt, dieses Thema aber zu einem späteren Zeitpunkt (kurz) refokussiert - allerdings in einem anderen Kontext. Mit Blick auf das gesamte Gespräch wird deutlich, dass hier der Aspekt der Platzierung von Emotionsthematisierungen zentral und zugleich interaktiv herausfordernd ist.

Beispiel 1 (vgl. zu den Transkriptionskonventionen Selting u.a. 2009) beginnt nach etwa zwei Minuten Gesprächszeit und ist in einer fortgeschrittenen Phase der Beschwerdenexploration situiert, in der die Ärztin mit einer Reihe aufeinanderfolgender Fragen auf die Eingrenzung des Problems abzielt. Der zitierte Ausschnitt zeigt, wie die Patientin Ängste im Zusammenhang mit den Schmerzen im Ellenbogen thematisiert (zum engen Zusammenhang von Schmerzen und Emotionen vgl. auch z. B. Spranz-Fogasy/Becker, in diesem Band), worauf die Ärztin nicht eingeht, sondern die im Vorfeld von ihr erfragte Schmerzcharakteristik refokussiert:

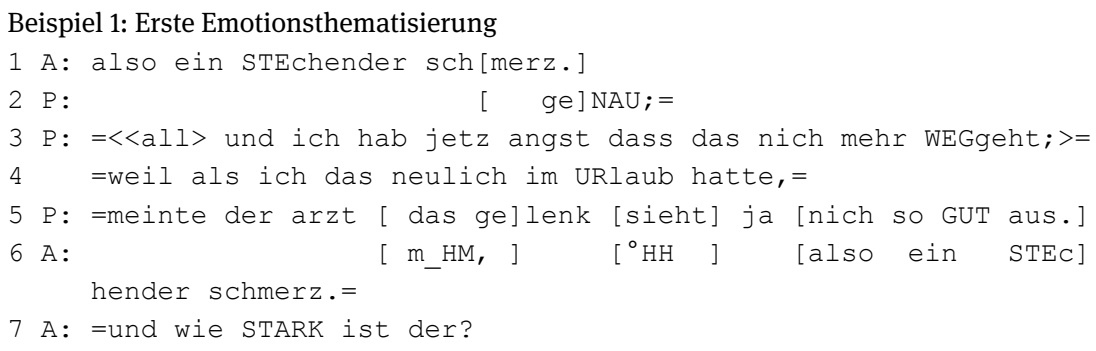

Kurz vor dem zitierten Ausschnitt fragt die Ärztin nach der Art des Schmerzes, den die Patientin im Ellenbogen verspürt, woraufhin diese den Schmerz als „so FIES;=irgendwie wie mit nem MESser;“ umschreibt (oben nicht zitiert). Die Ärztin schlägt für diese Charakterisierung den Begriff „STEchender schmerz“ vor (Z.1), was die Patientin in terminaler Überlappung mit „geNAU“ bestätigt (Z. 2). Mit schnellem Anschluss führt die Patientin einen neuen Aspekt ihrer Beschwerden ein: ihre Angst davor, dass der Schmerz „nich mehr WEGgeht“ (Z.3). 
Diese explizite Emotionsthematisierung nimmt die Ärztin zunächst nicht erkennbar entgegen, weder durch (zustimmende) Rückmeldesignale noch z. B. nonverbal durch Nicken oder eine mimische Aufnahme (Z. 3 f.). In Z. 6 platziert sie schließlich in kurzem Abstand ein Rezeptionssignal, ein deutliches Einatmen sowie eine recht früh platzierte Turnübernahme, mit der sie die zuvor interaktiv als stechend charakterisierte Schmerzqualität (Z.1f.) refokussiert; daran fügt sie mit schnellem Anschluss eine Frage zur Schmerzstärke an und setzt somit das Erfragen der Schmerzcharakteristika fort (Z.7).

Indem sie die Angstthematisierung nicht erkennbar entgegennimmt, bereits in der Äußerungsmitte der Patientin eine Turnübernahme einleitet und dann tatsächlich früh durchführt sowie die zuvor thematisierte Schmerzqualität unkommentiert wieder aufnimmt, stuft die Ärztin die Emotionsthematisierung in ihrer Relevanz herunter. Zugleich wird sie gleichsam aus dem Gesprächsverlauf „gelöscht“; sie wird, mit Jefferson, „sequentially deleted“ (Jefferson 1978, 229).

Kurz darauf thematisiert die Patientin erneut ihre Angst, dass der Schmerz sich als chronisch erweisen könnte. Auch diese Emotionsthematisierung nimmt die Ärztin nicht auf, sondern leitet stattdessen zur Untersuchung des Gelenks über:

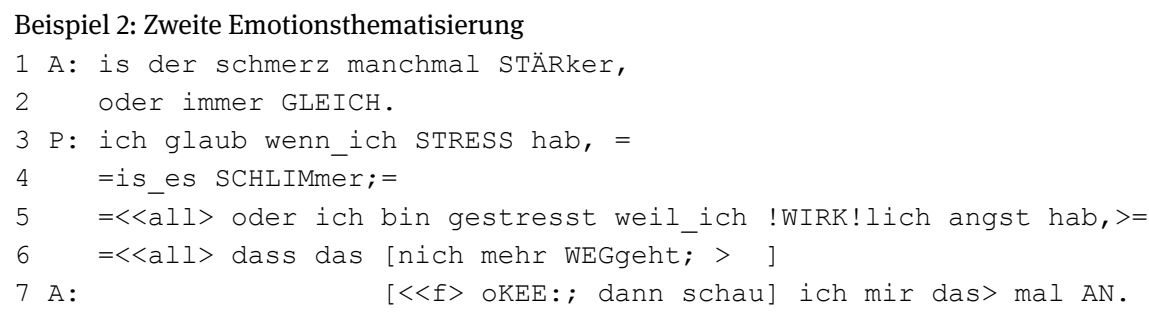

Auf die Frage der Ärztin nach der Schmerzstärke antwortet die Patientin mit einer subjektiven Theorie (,ich glaub“, Z. 3), nach der die Stärke des Schmerzes in Abhängigkeit von ihrem persönlichen Stresserleben variiere, was möglicherweise mit ihrer Angst vor einer Chronifizierung des Schmerzes zusammenhänge (Z.3-6). Mit dem stark betonten Adverb „wirklich“ stuft sie ihre negative Emotion zusätzlich hoch (Z.5). Sie stellt hier also einen kausalen Zusammenhang zwischen ihrer Angst und der Schmerzstärke her und gibt der Ärztin auf diese Weise einen - potentiell wichtigen - Einblick in ihre subjektive Theorie über die Aufrechterhaltung/Verschlechterung ihrer Schmerzen (zu subjektiven Krankheitstheorien vgl. ausführlich Birkner 2006; Birkner/Vlassenko, in diesem Band).

Die Ärztin geht auf diesen Aspekt des Krankheitswissens der Patientin nicht ein: Auch diese erneute Thematisierung von Angst nimmt sie nicht erkennbar entgegen und übernimmt vielmehr bereits zu einem frühen Zeitpunkt mit einem abschließenden „oKEE:;“ den Turn, mit dem sie dann zur körperlichen Untersuchung und somit zu einer neuen Gesprächsphase überleitet. Auch die zweite Emotionsthematisierung wird also nicht aufgenommen, sondern in ihrer Relevanz heruntergestuft. 
Interessant ist nun, dass die Ärztin das Thema Angst kurz darauf (ca. zwei Minuten später) von sich aus rethematisiert: Während sie der Patientin ein Rezept ausstellt, fragt sie: „und sie sind zur zeit allgemein recht geSTRESST; Oder?“. Die Patientin bestätigt diese Vermutung und stuft diesen Thematisierungsversuch zugleich in seiner Relevanz herunter: „JA;=ZIEmlich;=aber naJA;“. Damit endet diese Sequenz, die Ärztin hakt an dieser Stelle nicht noch einmal nach. Sie hat also die ersten Thematisierungsversuche zwar zunächst nicht aufgenommen, das emotionale Erleben der Patientin aber offensichtlich lediglich zugunsten anderer Themen zurückgestellt, um es später erneut anzusprechen. Dieses Vorgehen kündigt sie allerdings nicht (vorstrukturierend) an, so dass die Patientin erst im Moment der Wiederaufnahme wissen kann, dass ihre vorherigen Thematisierungsversuche nicht ignoriert, sondern lediglich zurückgestellt wurden. Hinzu kommt, dass der zuvor von der Patientin etablierte Zusammenhang zwischen Angst und Schmerzstärke in der Rethematisierung der Ärztin verlorengeht. Dieser möglicherweise wichtige Aspekt des Patientenwissens bleibt somit durch die verspätete Reaktion der Ärztin unberücksichtigt.

Einerseits widerspricht dieses Zurückstellen der Emotionsthematisierung der Regel, dass Emotionen Vorrang in der Interaktion haben (vgl. Sacks 1992, 572). Dies gilt nicht nur für Alltagsgespräche, sondern aufgrund der besonderen Relevanz von Emotionen für das Krankheitserleben auch im stärker strukturierten institutionellen Arzt-Patient-Gespräch (vgl. Koerfer/Obliers/Köhle 2004); dies besonders aufgrund des flüchtigen Charakters von Emotionen, „die eben dort, wo sie im Gespräch thematisch werden, auch unmittelbar (,an Ort und Stelle') wahrzunehmen sind“ (Koerfer u. a. 2008, 54).

Andererseits laufen die Versuche der Patientin, ihre Emotionen zu thematisieren, gewissermaßen den ärztlichen Bemühungen entgegen, mit gezielten Fragen spezifische Schmerzaspekte zu fokussieren, da die Emotionsthematisierung mitten in diesen „Fragenkatalog“ der Ärztin hinein platziert wird. Was also auf den ersten Blick wie ein Ignorieren von Thematisierungsversuchen der Patientin aussieht, bekommt im Kontext des gesamten Gesprächs eine zweite interaktive Logik, die auf dem ärztlichen Bemühen um Gesprächsstrukturierung und Fokussierung der Beschwerden beruht.

Der hier in Ausschnitten abgebildete und in einer Vielzahl von Arzt-PatientGesprächen zu beobachtende relativ schematische Gesprächsablauf scheint zwar im Großen und Ganzen zum impliziten PatientInnenwissen zu gehören, da mehrheitlich eine patientenseitige Orientierung an dieser Struktur zu beobachten ist. Er scheint aber im Detail, d.h. mit seinen jeweils ganz unterschiedlichen Gesprächsaufgaben und -themen, für PatientInnen nicht immer transparent zu sein. Somit ist von PatientInnenseite auch nicht immer deutlich, dass Emotionsthematisierungen interaktiv nicht an jeder Stelle des Gesprächs gleich einfach zu realisieren sind: Während sie z. B. mit eher geringem interaktivem „Störungspotential“ in der offenen Phase der Beschwerdenschilderung platziert werden können, in der die PatientInnen selbst die Möglichkeit haben sollten darzulegen, was sie bewegt (vgl. z. B. Heritage/Robinson 2006, 89), erscheint die (im obigen Gespräch von der Patientin gewählte) Phase, in 
der das Gespräch von ärztlicher Seite stärker mit Fragen gelenkt wird, in Hinblick auf eine interaktiv reibungslose Emotionsthematisierung ungünstiger.

Es zeigt sich deutlich das häufig anzutreffende Spannungspotential zwischen „the ,voice of medicine“ and the ,voice of lifeworld““ (Mishler 1984, 14): Die Patientin stellt ihre Ängste in einen direkten Zusammenhang zu ihren Schmerzen und platziert ihre Emotionsthematisierungen folgerichtig mitten in die auf die Schmerzcharakterisierung ausgerichtete Beschwerdenexploration hinein. Die Ärztin hingegen behandelt die Angst der Patientin als zusätzliches Thema, das sie - aus dieser Perspektive ebenfalls folgerichtig - erst nach Abschluss der Schmerzthematik zu platzieren versucht.

\section{Formen der kommunikativen Darstellung von Angst}

In medizinischer Kommunikation besteht eine zentrale Aufgabe der Beteiligten darin herauszuarbeiten, welche der geschilderten Probleme tatsächlich einer Behandlung bedürfen, wenn ja von welcher Fachdisziplin, und welche der Probleme nicht behandlungsbedürftig sind, da es sich um „Alltagsprobleme“ handelt. Diese interaktive Aufgabe des Abgleichens von PatientInnen- und BehandlerInnenwissen ist besonders herausfordernd bei Erkrankungen, an denen die Emotion Angst beteiligt ist.

Angst gehört zu den basalen Emotionen und ist eine der am häufigsten und am stärksten erlebten Empfindungen. In klinischer Perspektive werden eine Vielzahl von Ängsten unterschieden, die in diagnostischen Manualen definiert werden. Sowohl ÄrztInnen als auch PatientInnen orientieren sich im Gespräch deutlich - wenn auch nicht unbedingt mit demselben Ergebnis - an den Angstkategorien dieser Manuale, wodurch diese Kategorien für die Interaktion relevant werden. Die interaktive Aufgabe der Kategorisierung von Angst gestaltet sich jedoch häufig schwierig: Obwohl Angsterkrankungen, die zu den häufigsten psychischen Störungen gehören, heutzutage als gut behandelbar gelten, ist ihr Verlauf oft chronisch, da sie im Durchschnitt erst nach fünf bis fünfzehn Jahren diagnostiziert werden.

Hier kann die Analyse der Art und Weise von Emotionsdarstellungen in interdisziplinärer Zusammenarbeit zu einer Verbesserung der Differenzialdiagnostik beitragen. Der Ansatz der linguistischen Differenzialtypologie wurde insbesondere von Elisabeth Gülich und Martin Schöndienst im Rahmen des Projektes „Linguistische Differentialtypologie epileptischer und anderer anfallsartiger Störungen“ entwickelt. Das Projekt wurde in Kooperation zwischen der Universität Bielefeld und dem Epilepsiezentrum Bethel durchgeführt und 1999-2001 von der DFG gefördert (für weitere Informationen siehe http://www.uni-bielefeld.de/lili/ personen/eguelich/Epiling. html). Dieses Vorhaben befasst sich mit der vergleichenden Analyse der Methoden, 
die die Beteiligten zur kommunikativen Darstellung unterschiedlicher Formen von Anfallsereignissen einsetzen. Auf diese Weise konnten eine Reihe linguistischer Merkmale identifiziert werden, die sich als differenzialdiagnostisch relevant für die Unterscheidung epileptischer und dissoziativer Anfälle erwiesen haben (vgl. für einen Überblick über zentrale Ergebnisse Surmann 2005, 16-20; vgl. auch Reuber/Ekberg, in diesem Band). In der interdisziplinären Kooperationsgruppe „Kommunikative Darstellung und klinische Repräsentation von Angst“ wurde anschließend speziell die kommunikative Darstellung von Angst im Zusammenhang mit verschiedenen neurologischen und psychiatrischen Erkrankungen untersucht. Diese Forschungsgruppe arbeitete 2004 am Zentrum für interdisziplinäre Forschung (ZiF) der Universität Bielefeld (für weitere Informationen siehe http://www.uni-bielefeld.de/lili/personen/eguelich/Angst.html). Sowohl das Projekt „Linguistische Differentialtypologie“ als auch das Vorhaben „Kommunikative Darstellung und klinische Repräsentation von Angst“ werden bis heute fortgesetzt.

Ein Befund dieser beiden Projekte war, dass die kommunikative Darstellung des Krankheitserlebens sich zum Teil deutlich unterscheidet, je nachdem, ob die InteraktantInnen die Emotion als Alltagsangst, Panikattacke oder als Angst im Zusammenhang mit (epileptischen) Anfällen einordnen.

Die im Folgenden präsentierten Beobachtungen zur Differenzialtypologie von Angst nehmen ihren Ausgangspunkt bei einer Reihe von Beobachtungen aus diesen beiden Forschungsprojekten (vgl. Gülich 2007; Gülich u. a. 2005; Gülich/CouperKuhlen 2007; Gülich/Lindemann 2010; Gülich/Lindemann/Schöndienst 2010; Schöndienst/Reuber 2008; Schöndienst/Lindemann 2012; Schwabe 2006, 252-280; Surmann 2005, 325-330), die in Lindemann (2012) zueinander in Beziehung gesetzt und erweitert wurden. Dadurch beruhen die unten sehr kurz zusammengefassten Ergebnisse auf der vergleichenden Analyse von mehr als 100 medizinischen Gesprächen, in denen die TeilnehmerInnen über Angst im Zusammenhang mit epileptischen und dissoziativen Anfällen, über Panikattacken sowie „alltägliche“ Ängste sprechen. So konnten kommunikative Merkmalsbündel herausgearbeitet werden, mit denen die Sprecher eine Typologisierung verschiedener Ängste vornehmen. Die folgende Tabelle gibt einen ersten Überblick über die Charakteristika der unterschiedlichen Angstdarstellungen: 
Tab. 1: Kommunikative Merkmale der Angstdifferenzierung (nach Lindemann 2012, 173)

\begin{tabular}{|c|c|c|c|}
\hline & Alttagsangst & Panik & Epileptische Angst \\
\hline Objekt/Auslöser & wird genannt & $\begin{array}{l}\text { nicht genannt, } \\
\text { relevant gesetzt }\end{array}$ & $\begin{array}{l}\text { nicht genannt, nicht } \\
\text { relevant gesetzt }\end{array}$ \\
\hline Plötzlichkeit & nicht relevant gesetzt & $\begin{array}{l}\text { sehr stark relevant } \\
\text { gesetzt }\end{array}$ & kaum relevant gesetzt \\
\hline Gefühlsqualität & keine Beschreibung & keine Beschreibung & $\begin{array}{l}\text { verstärkte Formulie- } \\
\text { rungsarbeit }\end{array}$ \\
\hline $\begin{array}{l}\text { „Unbeschreibbarkeit“ } \\
\text { (vgl. Gülich 2005) }\end{array}$ & nein & nein & ja \\
\hline Relevanzhochstufung & kaum & sehr stark & zum Teil \\
\hline Erlebensdomäne & Alltag & ambivalent & Fremdheit \\
\hline Rationalisierung & ja & nein & nein \\
\hline Normalisierung & ja & nein & nein \\
\hline Thematisierbarkeit & kontextabhängig & $\begin{array}{l}\text { als einfach } \\
\text { gekennzeichnet }\end{array}$ & $\begin{array}{l}\text { als schwierig } \\
\text { gekennzeichnet }\end{array}$ \\
\hline
\end{tabular}

Einige dieser Charakteristika werden in den folgenden Abschnitten anhand von Transkriptausschnitten erläutert. Aus Platzgründen können allerdings nur einzelne Merkmale der Darstellung von Alltagsangst und von Panik angesprochen werden (siehe daher für eine ausführliche Darstellung Lindemann 2012, 139-174). Die Beispiele stammen aus dem ca. 150 Gespräche umfassenden Korpus der Bielefelder Projekte (siehe S.163f.; für eine ausführliche Beschreibung dieses Korpus vgl. Lindemann 2012, 27-32).

\subsection{Alltagsangst}

Wenn Angst von den Beteiligten als alltäglich dargestellt wird, d. h. als nicht behandlungsbedürftiges Ereignis, dann wird dieses Erleben „als eine selbstverständliche und damit für das Gegenüber leicht nachvollziehbare Form der Angst ausgewiesen“ (Schwabe 2006, 278). Dafür werden sprachliche Verfahren der Normalisierung und Rationalisierung eingesetzt. Eine Rationalisierung der Angst kann z. B. erzielt werden, indem Gründe für das Auftreten der Angst oder Beispielsituationen angeführt werden. Normalisiert wird die Angst darüber hinaus durch Adverbien wie „natürlich“, „halt“, „eben“ etc. Die Beteiligten machen dadurch deutlich, „dass auch andere Personen ebenso gefühlt hätten, dass Angst also in diesen Situationen erwartbar ist“ (ebd.). Die Formulierungsarbeit bezieht sich somit auf die Begründung der Emotion gegenüber dem/der aktuellen InteraktionspartnerIn, wohingegen die Art und Weise 
des Angsterlebens nicht als erklärungsbedürftig ausgewiesen, sondern als grundsätzlich bekanntes Erleben vorausgesetzt wird (vgl. Lindemann 2012, 143; Schwabe 2006, 278). Auffallend ist zudem, dass die Angst grundsätzlich an einen Trigger und/oder ein Objekt gebunden wird (vgl. Lindemann 2012, 147-149).

Das folgende Beispiel ist typisch für eine solche Darstellung von Alltagsangst:

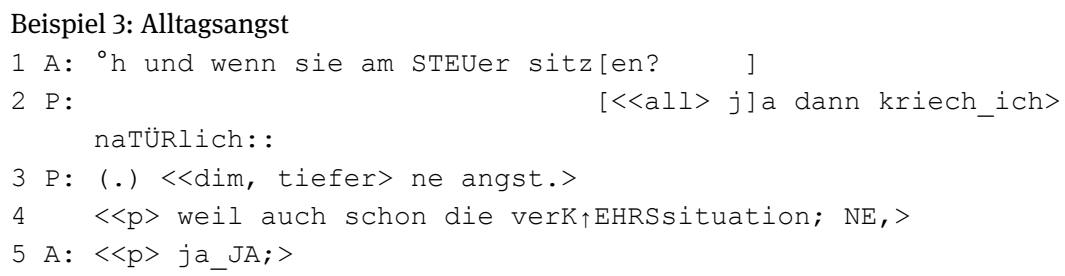

Der Arzt erfragt das Angstpotential beim Erleben epileptischer Anfälle während des Autofahrens und bringt dadurch einen möglichen Auslösefaktor für Angst in die Interaktion ein (,am STEUer sitzen“, Z.1). Darauf reagiert die Patientin bereits in terminaler Überlappung und mit hoher Sprechgeschwindigkeit mit Zustimmung: Wenn sie selbst am Steuer sitze und einen Anfall erlebe, empfinde sie Angst (Z.2f.). Das Auftreten von Angst kennzeichnet sie durch das betonte Adverb „naTÜRlich::“ als normal und unterstreicht diese Aussage durch die prosodische Gestaltung, mit der sie das Gesagte als etwas Selbstverständliches ausweist. An diese Turnkonstruktionseinheit fügt sie mit der kausalen Konjunktion „weil“ als weitere Begründung „die verK个EHRSsituation“ an (Z.4). Sie gibt also eine rationale Erklärung für ihre Angst. Auch diese Aussage markiert sie prosodisch als etwas Normales, Selbstverständliches. In tag-position fordert sie eine interaktive Bestätigung ihrer Aussage, indem sie ein betontes „NE,“ anfügt, auf das der Arzt ohne Verzögerung mit Zustimmung reagiert.

Die Patientin macht deutlich, dass es ihr wichtig ist, die Gründe für ihre Angst von ihrem Interaktionspartner als normal und rational ratifiziert $\mathrm{zu}$ wissen, und dieser bestätigt wiederum die Normalität oder Erwartbarkeit ihrer Angst. Eine Beschreibung der Gefühlsqualität gibt die Patientin weder in dieser Sequenz (die ja eher auf die situative Begründung der Angst ausgerichtet ist) noch an anderen Stellen des Gesprächs. Das Gefühl an sich wird somit sowohl von ihrer Seite als auch vonseiten des Arztes als keiner Erklärung bedürftig behandelt. Die Angst scheint dadurch in ihrer Qualität intersubjektiv eindeutig zuzuordnen zu sein.

Diese Art der kommunikativen Darstellung von Angst wird differenziert von Schwabe (2006, 266-280) beschrieben. In dem von ihr untersuchten Korpus tauchen diese Verfahren bei der Beschreibung von Angsterlebnissen auf, die eine „Reaktion auf die Anfälle“ darstellen (ebd., 266). Durch die Untersuchung eines Korpus, in dem ganz unterschiedliche Arten von Ängsten thematisiert werden (vgl. Lindemann 2012, 139-174), wird jedoch deutlich, dass diese kommunikativen Mittel nicht auf die Darstellung von Angst als Reaktion auf Anfallsereignisse beschränkt sind; sie werden 
vielmehr allgemein für die Darstellung alltäglicher Angst verwendet, die von den Beteiligten als nicht behandlungsbedürftiges Phänomen eingestuft wird.

\subsection{Panik}

Für die Kommunikation von Panikattacken ist vor allem die Betonung der Plötzlichkeit der Attacken und ihrer Unabhängigkeit von erkennbaren Auslösern charakteristisch. Damit geht einher, dass die PatientInnen nicht versuchen, ihre Angst als „normal“ und rational erklärbar darzustellen. Vielmehr stufen sie deren Intensität nahezu durchgehend extrem hoch, häufig sprechen sie sogar von Todesangst. Dabei machen sie vielfach von vorgeformten Strukturen, wie z. B. „es ging gar nichts mehr“, Gebrauch (vgl. dazu ausführlich Gülich 2007; Knerich 2012).

Trotzdem wird die Art und Weise des Gefühls nicht als andersartig ausgewiesen, wie dies insbesondere bei der Kommunikation epileptischer Angst zu beobachten ist; es treten kaum Formulierungsschwierigkeiten oder Verzögerungen in der Thematisierung auf. Vielmehr bringen die Betroffenen ihre Attacken meist selbstinitiiert und spontan zur Sprache und es ist immer wieder zu beobachten, dass sie dieses Thema auch entgegen interaktiver Zugzwänge oder beim Versuch der Turnübernahme durch den/die GesprächspartnerIn beibehalten. Die PatientInnen beschreiben die Attacken häufig dergestalt, dass sie verschiedenste Symptome in (langen) Listen aufzählen, ohne jedoch Erläuterungen der Gefühlsqualität zu geben. Im Zuge dieser Symptome, häufig dahinter versteckt, nennen sie ihre Angst (vgl. Schöndienst/Lindemann 2012, 66).

Das folgende Beispiel, in dem die Patientin ihr subjektives Erleben während einer Angsterkrankung beschreibt, zeigt mehrere dieser Charakteristika der Darstellung von Panik:

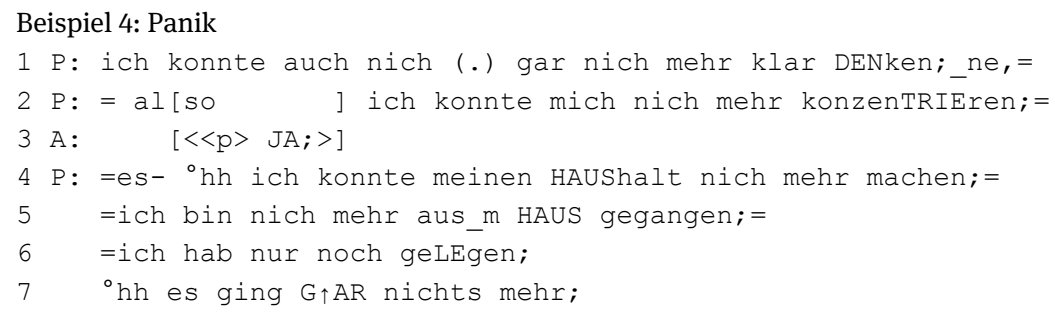

Die Patientin stuft die erlebten Symptome, die sie in Form einer mehrteiligen Liste sowie in Form absoluter Verneinungen ihrer alltäglichen „Funktionsfähigkeit“ präsentiert (,gar nich mehr“, „nich mehr“, „nich mehr“, „nich mehr“, „nichts mehr“), stark hoch. Dadurch hebt sie die besondere Einschränkung durch die Attacken hervor und gibt durch „nich mehr“ zugleich einen Hinweis darauf, dass diese Einschränkung zuvor nicht bestanden hatte. Ihre Darstellung abschließend nutzt sie die für sie indi- 
viduell typische, im Gespräch mehrfach genutzte vorgeformte Struktur „es ging gar nichts mehr“, mit der sie summarisch die Einschränkung durch die Attacken sowie die Stärke der Symptome maximal hochstuft.

Die in diesem Beispiel zu beobachtende Betonung der Stärke der Panik, der daraus resultierenden Einschränkung sowie die Auflistung von Symptomen sind (neben den weiteren in Tabelle 1 genannten Charakteristika) durchgängige Darstellungsmerkmale im Gespräch mit dieser Patientin. Ähnliche Sequenzen finden sich an vielen Stellen in diesem Gespräch, aber ebenso auch in Gesprächen mit anderen PatientInnen; sie sind typisch für die interaktive Typologisierung des Angsterlebens als Panik.

\section{Diskussion}

Emotionen und Emotionsthematisierungen gelten einerseits sowohl auf PatientInnen- als auch auf BehandlerInnenseite als relevanter Faktor für Erleben, Behandlung und Bewältigung von Erkrankungen. Zudem, das zeigen gesprächsanalytische Arbeiten, können sie wichtige Hinweise für Differenzialdiagnostik und -therapie liefern. Andererseits zeigt die empirische Realität, dass Emotionsthematisierungen von allen Beteiligten als interaktiv schwierige oder sogar gemiedene Gesprächsaufgabe behandelt werden. Diese unterschiedlichen Facetten von Emotionen in medizinischer Kommunikation wurden in den Kapiteln 3 und 4 exemplarisch verdeutlicht.

In Kapitel 3 wurden Beispiele präsentiert, in denen die Emotionsthematisierung für die Beteiligten zur interaktiven Herausforderung wird: Die patientenseitig zweimalig initiierte Thematisierung von Angst vor der Chronifizierung von Schmerzen wird von der Ärztin zunächst nicht aufgegriffen, dann aber zu einem späteren Zeitpunkt erneut fokussiert. Es wird deutlich, dass sich die beiden in diesem Gespräch einander entgegenlaufenden kommunikativen Aufgaben der Ärztin - einerseits dem Primat der Emotion zu folgen und die Thematisierungsbemühungen der Patientin ernst zu nehmen, andererseits der von dieser scheinbar betriebenen Defokussierung der Schmerz-Charakteristika entgegenzuwirken - nur schwer miteinander vereinbaren lassen. Eine mögliche Lösung, die zudem das interaktive Spannungspotential des zweimaligen Übergehens von Emotionsthematisierungen verringern würde, könnte (nicht nur in diesem Fall, sondern grundsätzlich) darin bestehen, das ärztliche Wissen um die angestrebte Gesprächsstrukturierung, d. h. die mit verschiedenen Gesprächsphasen verbundenen Gesprächsaufgaben und -themen, mit den PatientInnen zu teilen und diesbezüglich (mehr) Transparenz herzustellen. Lokal könnte dies bedeuten, das zeitweise Zurückstellen einer Emotionsthematisierung metadiskursiv zu kommentieren sowie eine Wiederaufnahme anzukündigen. Bezüglich der globalen Gesprächsstruktur könnten zudem metadiskursive Überleitungen zwischen einzelnen Gesprächsphasen mit Hinweisen auf jeweils relevante Gesprächsaufgaben 
den PatientInnen wichtige Orientierung und zugleich mehr Mitgestaltungsmöglichkeiten geben.

In Kapitel 4 wurde am Beispiel der Emotion Angst gezeigt, welches differenzialtypologische Potential die gesprächsanalytische Untersuchung von Emotionsthematisierungen haben kann. Eine Herausforderung bei der Diagnostik von Angst besteht darin, dass Ärzte zur Einschätzung der Angst vor allem auf Beschreibungen durch die Betroffenen, auf einen Zugang zu deren Erfahrungswissen angewiesen sind, zumal von außen beobachtbare Begleitphänomene wie Zittern oder Vermeidungsverhalten etc. häufig nicht zugänglich sind. Daher spielt das diagnostische Gespräch hier eine zentrale Rolle. Verzögerungen bei der Diagnostik von Angsterkrankungen können somit auch mit der Schwierigkeit zusammenhängen, Angst zu kommunizieren. Da Angststörungen hauptsächlich im Gespräch diagnostiziert und behandelt werden, ist es umso erstaunlicher, dass über konkrete Formen der kommunikativen Darstellung von Angst noch wenig bekannt ist. Die Gesprächsforschung liefert hier wichtige Erkenntnisse über die kommunikativen Verfahren, mit denen die Beteiligten Angst als alltägliches, nicht behandlungsbedürftiges Phänomen, als Panik oder als Symptom eines epileptischen Anfalls darstellen.

Bezüglich gesprächsanalytischer Ansätze linguistischer Differenzialtypologie von Emotionen ist darauf hinzuweisen, dass diese Erkenntnisse auf der Analyse der unterschiedlichen Perspektiven basieren, die die GesprächsteilnehmerInnen einander vermitteln. Daraus folgt, dass sich nur insofern Rückschlüsse auf medizinische Kategorien ergeben, als ersichtlich wird, als was die TeilnehmerInnen die thematisierten Ängste behandeln. So kann z. B. eine bestimmte Angst von dem einen Interaktanten kommunikativ als eine übermäßige, behandlungsbedürftige Angst dargestellt werden, während die andere sie als eine „normale“, alltägliche Angst einordnet. Welches der beiden Wissens- oder Zuordnungssysteme in einem solchen Fall von den TeilnehmerInnen als das „richtige“ und somit gültige für die aktuelle Interaktionssituation gekennzeichnet wird, wird in manchen Fällen als durch den institutionellen Rahmen vorgegeben behandelt, in anderen wird es mehr oder weniger explizit ausgehandelt.

Insgesamt steht die Untersuchung von Emotionen in medizinischer Kommunikation trotz bereits vielfältiger Ergebnisse noch eher am Anfang bzw. wird insbesondere in der Gesprächsforschung noch wenig systematisch betrieben. Die stärkere Fokussierung emotionaler Anteile könnte aber nicht nur allgemein zu einem besseren Verständnis medizinischer Kommunikation beitragen; in der Praxis könnte - durch das Aufheben der Diskrepanz zwischen theoretisch vorhandenem Wissen um die Relevanz von Emotionen und der Relevanzrückstufung im konkreten Gespräch - eine qualitative Verbesserung medizinischer Kommunikation erzielt werden:

The rewards of improving clinicians' emphatic response to patients are potentially great: improved relationships, better quality of care, and greater satisfaction for patients and clinicians alike. (Suchman u. a. 1997, 682) 


\section{Literatur}

Beach, Wayne A. u. a. (2005): Disclosing and responding to cancer "fears" during oncology interviews. In: Social Science \& Medicine 60, 893-910.

Bergmann, Jörg (1992): Veiled morality: notes on discretion in psychiatry. In: Paul Drew/John Heritage (Hg.): Talk at Work. Cambridge, 137-162.

Birkner, Karin (2006): Subjektive Krankheitstheorien im Gespräch. In: Gesprächsforschung - OnlineZeitschrift zur verbalen Interaktion 7, 152-183.

Birkner, Karin/Ivan Vlassenko (in diesem Band): Subjektive Theorien von Krankheit und Gesundheit. Buser, Kurt/Thomas Schneller/Klaus Wildgrube (2007): Kurzlehrbuch Medizinische Psychologie Medizinische Soziologie. München.

Fiehler, Reinhard (1990): Erleben und Emotionalität als Problem der Arzt-Patienten-Kommunikation. In: Konrad Ehlich u. a. (Hg.): Medizinische und therapeutische Kommunikation. Opladen, 41-65.

Fiehler, Reinhard (2001): Emotionalität im Gespräch. In: Klaus Brinker u. a. (Hg.): Text- und Gesprächslinguistik, Band II. Berlin/New York, 1425-1438.

Fiehler, Reinhard (2005): Erleben und Emotionalität im Arzt-Patienten-Gespräch. In: Mechthild Neises/Susanne Ditz/Thomas Spranz-Fogasy (Hg.): Psychosomatische Gesprächsführung in der Frauenheilkunde - ein interdisziplinärer Ansatz zur verbalen Intervention. Stuttgart, 120-136.

Gülich, Elisabeth u. a. (2005): Ictal fear versus panic attacks: first results from conversation analysis of clinical interviews. In: Epilepsia 46, 343.

Gülich, Elisabeth (2005): „Unbeschreibbarkeit“: Rhetorischer Topos - Gattungsmerkmal Formulierungsressource. In: Gesprächsforschung - Online-Zeitschrift zur verbalen Interaktion 6, 222-244.

Gülich, Elisabeth (2007): „Volle Palette in Flammen“. Zur Orientierung an vorgeformten Strukturen beim Reden über Angst. In: Psychotherapie und Sozialwissenschaft 9, 59-87.

Gülich, Elisabeth/Elizabeth Couper-Kuhlen (2007): Zur Entwicklung einer Differenzierung von Angstformen im Interaktionsverlauf: Verfahren der szenischen Darstellung. In: Reinhold Schmitt (Hg.): Koordination: Beiträge zur Analyse multimodaler Interaktion. Tübingen, 293-337.

Gülich, Elisabeth/Katrin Lindemann (2010): Communicating emotion in doctor-patient interaction: a multidimensional single-case analysis. In: Dagmar Barth-Weingarten/Elisabeth Reber/Margret Selting (Hg.): Prosody in Interaction. Amsterdam, 269-294.

Gülich, Elisabeth/Katrin Lindemann/Martin Schöndienst (2010): Interaktive Formulierung von Angsterlebnissen im Arzt-Patient-Gespräch. Eine Einzelfallstudie. In: Ulrich Dausendschön-Gay/Christine Domke/Sören Ohlhus (Hg.): Wissen in (Inter-)Aktion. Berlin, New York, 135-160.

Heritage, John/Anna Lindström (2012): Knowledge, empathy, and emotion in a medical encounter. In: Anssi Peräkylä/Marja-Leena Sorjonen (Hg.): Emotion in Interaction. Oxford u. a., 256-273.

Heritage, John/Jeffrey D. Robinson (2006): The structure of patients' presenting concerns: physicians' opening questions. In: Health Communication 19, 89-102.

Jefferson, Gail (1978): Sequential aspects of story telling in conversation. In: Jim N. Schenkein (Hg.): Studies in the Organization of Conversational Interaction. New York, 213-248.

Jefferson, Gail (1988): On the sequential organization of troubles-talk in ordinary conversation. In: Social Problems 35, 418-441.

Knerich, Heike (2012): Vorgeformte Strukturen als Formulierungsressource beim Sprechen über Angst und Anfälle. Dissertation. Dortmund. 
Koerfer, Armin u. a. (2008): Training und Prüfung kommunikativer Kompetenz. Aus- und Fortbildungskonzepte zur ärztlichen Gesprächsführung. In: Gesprächsforschung - OnlineZeitschrift zur verbalen Interaktion 9, 34-78.

Koerfer, Armin/Rainer Obliers/Karl Köhle (2004): Emotionen in der ärztlichen Sprechstunde. Förderung empathischer Kommunikation durch Fortbildung in psychosomatischer Grundversorgung. In: Psychotherapie und Sozialwissenschaft 6, 243-262.

Lalouschek, Johanna (1993): „Irgendwie hat man ja doch bißl Angst“. Zur Bewältigung von Emotionen im psychosozialen ärztlichen Gespräch. In: Petra Löning/Jochen Rehbein (Hg.): Arzt-Patienten-Kommunikation. Zur Bewältigung von Emotionen im psychosozialen ärztlichen Gespräch. Berlin, New York, 177-190.

Lindemann, Katrin (2012): Angst im Gespräch. Eine gesprächsanalytische Studie zur kommunikativen Darstellung von Angst. Göttingen.

Lindtner-Rudolph, Heide/Hubert J. Bardenheuer (in diesem Band): Sprache am Lebensende: Chancen und Risiken ärztlicher Gesprächsführung in der Palliativmedizin.

Lüdke, Christian/Peter Langkafel (2008): Breaking Bad News: Das Überbringen schlechter Nachrichten in der Medizin. Heidelberg.

Mishler, Elliot G. (1984): The Discourse of Medicine: Dialectics of Medical Interviews. Norwood.

Peräkylä, Anssi/Marja-Leena Sorjonen (Hg.) (2012): Emotion in Interaction. Oxford u. a.

Reuber, Markus/Katie Ekberg (in diesem Band): Das Potential linguistischer interaktionaler Beiträge zur Differentialdiagnose epileptischer und nicht-epileptischer Anfälle in der Neurologieambulanz.

Rockenbauch, Katrin/Oliver Decker/Yve Stöbel-Richter (2006): Kompetent kommunizieren in Klinik und Praxis. Lengerich u. a.

Ruusuvuori, Johanna (2013): Emotion, affect and conversation. In: Jack Sidnell/Tanya Stivers (Hg.): The Handbook of Conversation Analysis. Malden/Oxford, 330-349.

Sacks, Harvey (1992): Lectures on Conversation. Band 2. Cambridge.

Schöndienst, Martin/Katrin Lindemann (2012): Panic, ictal fear and hyperventilation. In: Markus Reuber/Steven C. Schachter (Hg.): Borderland of Epilepsy Revisited. New York, 63-73.

Schöndienst, Martin/Markus Reuber (2008): Epilepsy and anxiety. In: Steven C. Schachter/Gregory L. Holmes/Kastelijn-Nolst Trenité, Dorothée G. A. (Hg.): Behavioral Aspects of Epilepsy. New York, 219-226.

Schwabe, Meike (2006): Kinder und Jugendliche als Patienten. Eine gesprächsanalytische Studie zum subjektiven Krankheitserleben junger Anfallspatienten. Göttingen.

Selting, Margret u. a. (2009): Gesprächsanalytisches Transkriptionssystem 2 (GAT 2). In: Gesprächsforschung - Online-Zeitschrift zur verbalen Interaktion 10, 353-402.

Sorjonen, Marja-Leena/Anssi Peräkylä (2012): Introduction. In: Anssi Peräkylä/Marja-Leena Sorjonen (Hg.): Emotion in Interaction. Oxford u. a., 3-15.

Spranz-Fogasy, Thomas/Maria Becker (in diesem Band): Beschwerdenexploration und Diagnosemitteilung im ärztlichen Erstgespräch.

Suchman, Anthony L. u. a. (1997): A model of empathic communication in the medical interview. In: Journal of the American Medical Association 8, 678-682.

Surmann, Volker (2005): Anfallsbilder: Metaphorische Konzepte im Sprechen anfallskranker Menschen. Würzburg. 